\title{
Rosiglitazone Protects Blood-Brain Barrier Integrity Following Ischemic Stroke by Reducing MMP-9 Expression Through a Caveolin-1-Dependent Pathway
}

\author{
Qianyi Huang \\ Second Xiangya Hospital \\ Wei Zhong \\ Second Xiangya Hospital \\ Xia Yi \\ Second Xiangya Hospital \\ Zhen Qin \\ Second Xiangya Hospital \\ Xiangqi Tang ( $\square$ txq6633@csu.edu.cn ) \\ Second Xiangya Hospital https://orcid.org/0000-0003-1709-4456
}

\section{Research}

Keywords: Rosiglitazone, Ischemic stroke, Caveolin-1, Blood-brain barrier, MMP-9

Posted Date: January 8th, 2021

DOI: https://doi.org/10.21203/rs.3.rs-139925/v1

License: (c) (i) This work is licensed under a Creative Commons Attribution 4.0 International License.

Read Full License 


\section{Abstract \\ Background}

Rosiglitazone (RSG) is a widely used antidiabetic drug which activates peroxisome proliferator-activated receptor-y. Recent work have shown that RSG can up-regulate caveolin-1 levels and ameliorate both chronic and acute brain injury. However, whether rosiglitazone can ameliorate ischemic injury in the brain via a caveolin-1-dependent pathway remains unknown.

\section{Methods}

Adult male sprague-dawley rats were randomly divided into sham operation group, model group, rosiglitazone group and rosiglitazone + daidzein group. The rat models of middle cerebral artery occlusion (MCAO) was established using the suture method, with ischemia for 2 hours and reperfusion for 22 hours. Neurological deficits were evaluated by the methods of Longa's standard scoring. Cerebral infarction volume was observed by staining with 2, 3, 5-triphenyltetrazolium chloride. Evans blue content reflects BBB permeability. The expressions of caveolin-1, matrix metalloproteinase-9(MMP-9) and occludin were detected by immunofluorescent staining and western blot.

\section{Results}

In this study, we found that the expression of caveolin-1 was increased in a rat model of stroke, and treatment with RSG significantly increased the levels of caveolin-1, reduced the release of MMP-9, and increased the expression of occludin. On the other hand, a caveolin-1 inhibitor daidzein canceled the protective roles of RSG in the MCAO model.

\section{Conclusions}

These data unveil that RSG might protect blood-brain barrier integrity by down-regulating the levels of MMP-9 via a caveolin-1-dependent pathway.

\section{Introduction}

Acute ischemic stroke (AIS) is a major cause of mortality and morbidity in the adult population worldwide[1]. Intensive basic and clinical studies have revealed multiple risk factors for stroke and clarified many mechanisms underlying acute ischemic injury. However, current treatment for AIS remains largely dependent on recombinant tissue plasminogen activator (rtPA)-mediated thrombolytic intervention or mechanical thrombectomy in appropriate patients. During and after AIS, blood-brain barrier (BBB) damage facilitates injury progression and increases the risk of haemorrhagic transformation, limiting the use of rtPA[2]. As for mechanical thrombectomy, just only a few percentages of patients meet the 
eligibility criteria[3]. Therefore, there is a continuous urgent need to explore potential therapeutic targets to improve neurological function following AIS.

Peroxisome proliferator-activated receptor-y (PPARY) is a member of the nuclear receptor superfamily, acting as a ligand-activated transcription factor[4]. Administration of PPAR-y agonists has been shown to protect against cerebral ischemia through multiple mechanisms, such as by inhibition of post-ischemic oxidative stress[5, 6], apoptosis[5], and ischemia-induced inflammation[6-9]. Recent in vitro and vivo research, Rosiglitazone (RSG), a PPARy agonist, was shown to increase caveolin-1 expression and protect against traumatic brain injury $[10,11]$. Caveolin-1 is a principal marker of caveolae which are invaginations of the plasma membrane[12]. Caveolin-1 has been shown to protect the integrity of BBB by decreasing matrix metalloproteases (MMPs), which degrade tight junctions during cerebral ischemia[13]. So, in this study, we investigated whether RSG can protect against AIS by modifying the expression of matrix metalloproteinase-9 (MMP-9) via a caveolin-1-dependent pathway.

In this study, we investigated the role played by RSG in the expression of caveolin-1. We also evaluated the effects of RSG on the levels of MMP-9 and occludin. Expression of caveolin- 1 was downregulated by intraperitoneally injected with a caveolin-1 inhibitor daidzein. BBB permeability and the expression of MMP-9 were monitored. The results showed that PPARy ligand RSG protected the integrity of BBB by reducing the level of MMP-9 via its ability to up-regulate caveolin-1.

\section{Methods}

\section{Ethics}

All experiments were performed in accordance with Guidelines for the Care and Use of Laboratory Animals formulated by the National Institutes of Health. The Second Xiangya Hospital Animal Care Committee of Central South University approved this study. Rats were housed in standard cages with ambient temperature of $22 \pm 1{ }^{\circ} \mathrm{C}$, humidity of $40-50 \%$, a 12-hour dark/light cycle, and free access to food and tap water.

\section{Animals and Groups}

Adult male Sprague-Dawley (SD) rats weighing 220 to $260 \mathrm{~g}$ were provided by the HUNAN SJA LABORATORY ANIMAL CO., LTD [license no. SCXK (Xiang) 2019-0004]. 96 SD rats were divided randomly into four groups: Sham group(24 rats), MCAO group (24 rats), RSG group(24 rats), and RSG + daidzein group (24 rats). RSG (Sigma-Aldrich) was dissolved in dimethyl sulfoxide (DMSO) and diluted to a final concentration of $2 \mathrm{mg} / \mathrm{mL}$. In the RSG group, rats received RSG immediately after MCAO at a dose of 10 $\mathrm{mg} / \mathrm{kg}$, q12 $\mathrm{h}$. In the RSG + daidzein group, intraperitoneal administration of Diadzein (Sigma-Aldrich) was given along with RSG immediately after the onset of stroke at a dose of $0.6 \mathrm{mg} / \mathrm{kg} .6$ rats in each group were used for TTC staining, 6 rats were used for Evans Blue staining in each group, 6 rats in each group were used for immunofluorescence staining, and 6 rats in each group were used for Western 
Blot.To compensate for dropouts, another 7 rats were enrolled to the study population, so there were 103 rats in total.

\section{Animal Model of MCAO}

Animal model of MCAO was established in SD rats as Longa et al. described[14]. Briefly, after the rats were injected with $10 \%(\mathrm{w} / \mathrm{v})$ chloral hydrate $(300 \mathrm{mg} / \mathrm{kg})$, a silicone-coated nylon monofilament with a heatblunted tip was passed through the bifurcation of the common carotid artery to the internal carotid artery until the tip reached the origin of the right middle cerebral artery. After 120 minutes of occlusion, the monofilament was withdrawn slowly to allow for 22 hours of reperfusion. The body temperature of rats should be maintained at $37 \pm 0.5^{\circ} \mathrm{C}$ until recovery. Rats in the sham operation group were subject to the same surgery except for the filament insertion.

\section{Neurological deficits score}

The neurological defects were assessed by two independent examiners who were blind to each experiment treatment before the killing[14]. 0: no symptoms of neurological deficits; 1 : left forepaws cannot be fully extended ; 2 : circling or rotating to the contralateral side; 3 : falling to the contralateral side; 4: No spontaneous activity with depressed levels of consciousness. Those with a score of 1-3 were included in the experiment, and those who were not satisfied were excluded. Other excluded conditions included subarachnoid hemorrhage, and rats died within 24 hours.

\section{Triphenyltetrazolium chloride staining}

To assess the infarction areas, after the rats were fully anesthetized, they were sacrificed by rapid cervical dislocation. Then, the brain tissue were quickly harvested and placed in a refrigerator at $-20^{\circ} \mathrm{C}$ for 20 minutes. Then we removed the olfactory bulb and cerebellum, and cut the brain tissue into 5 continuous coronal sections with a thickness of $2 \mathrm{~mm}$. The sections were incubated with 1\% (w/v) 2,3,5triphenyltetrazolium chloride (TTC; Sigma) in the dark at $37^{\circ} \mathrm{C}$ for 30 minutes and were flipped every 10 minutes. Then the brain slices were fixed in $4 \%(\mathrm{w} / \mathrm{v})$ paraformaldehyde solution at $4^{\circ} \mathrm{C}$ overnight. Pictures were taken and infarct volume was calculated with Image J software (ImageJ, MD, USA).

\section{Evans Blue extravasation}

Evans blue (EB; Sigma) was dissolved in saline and rats were injected with $2 \% \mathrm{~EB}(4 \mathrm{ml} / \mathrm{kg})$ via the tail vein. After 2 hours, rats were perfused with $0.9 \%(\mathrm{w} / \mathrm{v})$ sodium chloride to flush away the intravascular dye from the blood vessels. Then the nonischemic and ischemic brain tissues were quickly harvested, weighed, and homogenized and proteins were precipitated with formamide solution at $60^{\circ} \mathrm{C}$ for 24 hours. After centrifuging at $5000 \mathrm{~g}$ for 5 minutes, the absorbance at $620 \mathrm{~nm}$ was measured with a multifunctional microplate reader (Eppendorf, Germany) in the supernatant. The extravasation of EB was evaluated with a standard curve and expressed as microgram per gram of brain tissue.

\section{Immunofluorescence staining}


At 24 hours after MCAO, the brains were removed after perfusion with $0.9 \%$ sodium chloride and $4 \%$ paraformaldehyde. Then the brain tissue were immersed in $4 \%$ paraformaldehyde solution for post fixation, dehydrated in gradient sucrose solutions of $15 \%$ and $30 \%$ at $4{ }^{\circ} \mathrm{C}$, embedded in optimal cutting temperature compound and cut into $20 \mu \mathrm{m}$ thick serial coronal sections. Sliced tissues were then blocked with buffer containing $1 \%$ goat serum, and $0.2 \%$ Triton in PBS and incubated with the following primary antibodies: rabbit polyclonal anti-MMP-9 (1: 500, Abcam), rabbit polyclonal anti-caveolin-1 (1: 500, Abcam), rabbit monoclonal anti-occludin antibody (1:100, Abcam), and rabbit monoclonal anti-CD31 antibody (1:100, Abcam). After overnight incubation at $4{ }^{\circ} \mathrm{C}$, sections were washed four times, secondary fluorescent antibodies were added and incubated for 1 hour in the dark at room temperature, and the nuclei were counterstained with 4',6-diamidino-2-phenylindole (DAPI, Sigma-Aldrich). The sections were observed using a fluorescence microscope (Olympus, Japan). We used the Image $\mathrm{J}$ software to perform the anlysis. Six images of slides were obtained per groups. Images were converted into an 8-bit format, and an intensity threshold was set and kept constant for all images analyzed. Mean fluorescence intensities was calculated by dividing the fluorescence intensities by the area of outlined regions.

\section{Western Blot}

After 22 hours of reperfusion, rats in each group were intraperitoneally injected with $10 \%$ chloral hydrate. After deep anesthesia, the ischemic brain tissues were quickly removed and were homogenized with RIPA lysis buffer. The mixtures were incubated on ice for 30 minutes and then centrifuged for $15 \mathrm{~min}$ at $4^{\circ} \mathrm{C}$. After collecting the supernatant and denaturing it, equal amounts of protein samples were electrophoretically seperated in $10 \%$ sodium dodecyl sulfate-polyacrylamide (SDS-PAGE) gels and then transferred to polyvinylidene fluoride (PVDF) membranes. PVDF membranes were placed in an incubation box containing $5 \%$ BSA and blocked for $2 \mathrm{~h}$ at room temperature on a shaker. Then the membranes were incubated with primary antibodies against MMP-9 (1:5000, Abcam), caveolin-1 (1:5000, Abcam), and occludin (1:1000, Abcam) at $4^{\circ} \mathrm{C}$ overnight. After washing the membranes with tris-buffered saline and $0.1 \%$ Tween-20 (TBS-T) for $7 \mathrm{~min} \times 4$ times, they were incubated with the corresponding secondary antibodies (1:3000, Santa Cruz) at room temperature for 2 hours. Then membranes were washed four times with TBS-T for 7 minutes each, and were developed with the enhanced chemiluminescence (ECL) (Servicebio, China). The membranes incubated with GAPDH primary antibodies (1:4000, Sigma) were regared as the loading control. The bands were visualized using the Gel image analysis system (Bio-Rad, Italy) and quantified using the Image $\mathrm{J}$ software. The final results were standardized and expressed as the ratios of target proteins to GAPDH.

\section{Statistical analysis}

Data were presented as mean \pm SD. Statistical analysis was performed with GraphPad Prism software (version 8.3) and data were analyzed with one-way ANOVA or two-way ANOVA followed by Tukey's multiple comparisons test. Calculate the Pearson correlation coefficient between each index and perform a significant test. $P<0.05$ was considered statistically significant. 


\section{Results}

\section{TTC staining}

The cerebral infarct volume in each group was evaluated using TTC staining. No infarction was found in the sham-operated rats. The RSG group $(P<0.05)$ showed a significant reduction in the infarct volumes compared with the MCAO group. Compared with the RSG group, the RSG + daidzein group $(P<0.05)$ showed significant increases(Figure2).

\section{Neurological deficit score}

Neurological defects were evaluated and scored at $24 \mathrm{~h}$ following ischemia. Compared with the sham operation group, the MCAO model group had significantly higher neurological deficit scores; Compared with MCAO group, the rats treated with RSG showed significant reduced neurological deficit scores. In comparison to RSG intervention group, rats in RSG+daidzein group showed higher longa scores (Table.1, Figure1).

\section{BBB permeability}

To assess BBB permeability after ischemia, the content of Evans blue in brain tissue was measured at 24 $\mathrm{h}$ after ischemic stroke. In the sham operation group, rat brain tissues were almost free of Evans blue, while the model group showed more Evans blue in the ischemic hemisphere. The extravasation of Evans blue in contralateral hemisphere was significantly lower than that in the ischemic hemisphere of rats with cerebral ischemia. Compared with the MCAO group, RSG-treated group showed significantly reduced Evans blue content. The protective effect of RSG was abolished by downregulation of caveolin-1 (Figure3).

\section{The expression of caveolin-1, MMP-9 and occludin in each group detected by Western blot}

We used western blot to quantify the protein levels of caveolin-1, MMP-9 and occludin. The expression of caveolin-1 and MMP-9 proteins was increased in ischemic brains, while the expression of occludin was down-regulated after cerebral ischemia. RSG intervention significantly reduced the MMP-9 protein levels, but further increased the expression of caveolin-1 and occludin.To confirm that the protective effect of RSG on BBB damage was achieved by upregulating caveolin-1, we administered the caveolin-1 antagonist daidzein $(0.6 \mathrm{mg} / \mathrm{kg})$ along with RSG immediately after the onset of stroke through intraperitoneal injection.We found that daidzein treatment together with RSG reversed their expression. These results suggest that caveolin-1 upregulation by RSG may contribute to amelioration of the BBB disruption in stroke rats. (Figure4)

\section{The changes of caveolin-1, MMP-9 and occludin in each group detected by immunofluorescence assay}

We further performed immunofluorescence to detect the expression of cavoelin-1, MMP-9 and occludin in each group. The expression of occludin was quantified via colocalization with CD31. Compared with the 
sham group, occludin in the MCAO model group changed from a continuous linear structure to a discontinuous or even a spot-like structure, indicating that the tight junctions were damaged after ischemia-reperfusion injury. Compared with the model group, the RSG-treated group had better continuity of occludin. Compared with the sham group, the fluorescence intensity of MMP-9 and caveolin- 1 were significantly increased in the model group. After treatment with RSG, the fluorescence intensity of caveolin-1 was further increased, while that of MMP-9 was significantly decreased. Daidzein could cancel the effect of RSG on caveolin-1, MMP-9, and occludin. (Figure5) We also assessed the expression of cavoelin-1, MMP-9 and occludin in the contralateral hemisphere in each group, however it did not show significant changes.(figure S1)

\section{Correlation analysis}

The correlation analysis showed that the content of EB was positively correlated with the neurological score $(r=0.7187, P<0.0001)$, while the expression of occludin was negatively correlated with the neurological score $(r=-0.8529, P<0.0001)$. Although the levels of caveolin- 1 and MMP-9 were positively correlated with neurological scores, they were not statistically significant(caveolin-1 $r=0.3319, P=0.1131 ; M M P-9 r=0.3795, P=0.0674$ ). . figure $S 2$, figure S3)

\section{Discussion}

This study showed reduced Evans blue extravasation in RSG-treated rats compared with untreated rats suffering from ischemic brain injury, suggesting that prevention of BBB disruption is one of the protective mechanisms of RSG following cerebral ischemia. BBB is composed of endothelial cells and their linking tight junctions, pericytes, astrocytic endfeet, and extracellular matrix. It is crucial in the maintenance of the homeostatic microenvironment for normal neuronal function, pointing to the BBB as an important target for the attenuation of brain damage in stroke. Tight junction proteins have been generally believed to play an important role in regulating BBB permeability and function, and the decreased tight junction proteins have correlation with the compromised BBB integrity. Tight junction proteins comprise different proteins such as occludin, claudin-5, and ZO-1. In the present study, we demonstrated that RSG significantly increased the tight junction proteins expression after ischemic stroke, indicating that RSG could effectively protect BBB integrity via promoting tight junction expression. The relationship between MMP-9 and tight junction proteins has been clearly shown. Cerebral ischemic injury can lead to damage of BBB by degrading tight junction proteins through the up-regulation of MMP-9[17]. Reports have shown several agents that prevent the up-regulation of MMP-9 and improve the outcome of cerebral ischemia. In this study, we also showed the decrease in the expression of MMP-9 by RSG, suggesting that RSG can prevent ischemic brain damage by stabilizing the disrupted BBB via decreasing MMP-9 and increasing tight junction proteins. Our data are in agreement with a previous study which showed that RSG decreased the levels of MMP-9 after experimental stroke[5].

Caveolin-1 is essential for the formation of caveolae which generally distributed in endothelial cells, smooth muscle cells, skeletal myoblasts, adipocytes, and fibroblasts[18]. Caveolin-1 modulates multiple 
cellular functions such as cell proliferation, cellular senescence, lipid metabolism, cholesterol tracking, and signal transduction[19, 20]. In the brain, caveolin-1 is primarily expressed in endothelial cells, pericytes, and astroglial cells[21, 22], and caveolin-1 can regulate neuronal signaling and promote dendritic growth[23]. Changes in caveolin-1 can cause a series of changes in BBB permeability, neuroinflammation, oxidative stress, apoptosis, neurogenesis and angiogenesis[12]. Previous reports have suggested that caveolin-1 can regulate MMP-9. Compared with wild-type mice, caveolin-1(-/-) mice exhibited higher proteolytic activity and disruption of tight junction proteins, while re-expression of caveolin-1 showed the opposite effect[13]. RSG is a widely used antidiabetic drug which activates the PPAR- $y$. The activation of PPAR- $y$ is responsible for a beneficial effect in experimental models and has been evidenced at the different phases of cerebral ischemia[13]. PPAR- $\gamma$ agonists, including RSG also seem to reduce recurrent stroke and total related vascular events[24]. RSG treatment can minimize ischemic stroke through a variety of mechanisms, such as inhibiting inflammation, apoptosis and oxidative stress[25-27]. Studies have shown that RSG can increase the expression of caveolin-1 in cancer cells and macrophages[28, 29]. Recently, Zhao et al. provided evidence that RSG can protect brain tissue and BBB by increasing the level of caveolin-1 in diffuse axonal injury[10, 11]. However, whether RSG can play a protective role on BBB in animal models of cerebral ischemia/reperfusion injury via a caveolin-1-dependent pathway remains unknown, so we conducted this experiment.

In this study, we found that the level of caveolin-1 was increased in ischemic brains, and RSG further increased caveolin-1 expression. There have been some studies investigating the expression and potential role of caveolin-1 after cerebral ischemic stroke, but the results are inconsistent. Gu et al showed that caveolin-1 was reduced in cerebral ischemia/reperfusion injury[30], and the decrease in caveolin-1 was associated with increased MMP-9 activity and increased BBB permeability. Their previous studies found that the down-regulation of caveolin-1 may be related to the production of nitric oxide (NO) after ischemia-reperfusion injury[31], and a non-selective nitric oxide synthase inhibitor $N(G)$-nitro -L-arginine methyl ester (L-NAME) reduced the production of NO, retained the expression of caveolin-1, inhibited the activity of MMPs and reduced the permeability of BBB[30], suggesting that caveolin-1 may play a protective role. In addition, experimental studies have found that caveolin-1 can regulate nitric oxide synthase to inhibit the production of nitric oxide products[32,33]. However, Zhang et al found that caveolin-1 was increased after cerebral ischemia, and the neuroprotection effect of green tea polyphenols may be related to the down-regulation of caveolin-1[34]. Huang et al also showed that Cerebralcare Granule ameliorated BBB disruption caused by cerebral ischemia, which may be related to the inhibition of caveolin-1[35]. The discrepancy is probably related to different stroke models or detection condition.

To investigate whether the protective effect of RSG during cerebral ischemia was mediated by its caveolin-1 up-regulating effect, we administered both RSG and the caveolin-1 antagonist daidzein in the RSG + daidzein group. We found that the therapeutic effect of RSG on BBB was canceled in the presence of daidzein. Compared with the RSG group, the rats in the RSG + daidzein group showed increased neurological function score, increased cerebral infarction volume, and increased Evans blue content in ischemic hemisphere, indicating that caveolin-1 plays a key role in the RSG-afforded protection in AIS. Moreover, after the administration of daidzein, the level of caveolin-1 decreased, while the level of MMP-9 
increased, suggesting that caveolin-1 might play a protective role by inhibiting the expression of MMP-9. The results of our study are similar to that of a recent research conducted by Yang et al[36], they showed that the expression of caveolin-1 was up-regulated in MCAO-injured mice, and pretreatment with cystatin $C$ could further increased caveolin- 1 and decreased the enzymatic activity of MMP-9. The up-regulation of caveolin-1 during ischemia may be a protective compensatory response to prevent brain damage.The positive correlation between caveolin-1 expression and functional deficit may be related to the compensatory increase of caveolin-1 during ischemia.

\section{Conclusions}

In summary, RSG might protect the integrity of BBB in ischemia reperfusion injury by reducing the levels of MMP-9 via its ability to up-regulate caveolin-1.

\section{Abbreviations}

rosiglitazone (RSG); middle cerebral artery occlusion (MCAO);

matrix metalloproteinase-9(MMP-9);

acute ischemic stroke (AIS); recombinant tissue plasminogen activator (rtPA); Evans blue (EB)

blood-brain barrier (BBB); peroxisome proliferator-activated receptor-y (PPARY);

sprague-dawley (SD); 2,3,5-triphenyltetrazolium chloride (TTC);

\section{Declarations}

\section{Funding}

This work was supported by the National Natural Science Foundation of China (Grant no.81271298)

\section{Competing interests}

The authors declare no conflict of interest.

\section{Ethics approval}

The Second Xiangya Hospital Animal Care Committee of Central South University approved this study.

\section{Consent to participate}

Not applicable

\section{Consent for publication}


Not applicable

\section{Availability of data and material}

All data generated or analysed during this study are included in this published article.

\section{Authors' contributions}

Qianyi Huang participated in the design of the study, implemented animal experiments, drafted and revised the manuscript. Wei Zhong was responsible for animal experiments and statistical processing. Xia Yi provided technical support. Zhen Qin performed the statistical analysis and revised the manuscript. Xiangqi Tang supervised the study and revised the manuscript. All authors read and approved the final manuscript.

\section{References}

1. Global regional, and national burden of stroke, 1990-2016: a systematic analysisfor the Global Burden of Disease Study 2016. Global, regional, and national burden of stroke, 1990-2016: a systematic analysisfor the Global Burden of Disease Study 2016. The Lancet. Neurology. 2019;18(5):439 - 58. 'doi:'10.1016/S1474-4422(19)30034-1.

2. Wardlaw JM, Sandercock PAG, Berge E. Thrombolytic therapy with recombinant tissue plasminogen activator for acuteischemic stroke: where do we go from here? A cumulative meta-analysis. STROKE. 2003;34(6):1437-42.

3. Henderson SJ, Weitz JI, Kim PY. Fibrinolysis: strategies to enhance the treatment of acute ischemic stroke. Journal of thrombosis and haemostasis: JTH. 2018;16(10):1932-40. 'doi:'10.1111/jth.14215.

4. Ouk T, Potey C, Gautier S, Bastide M, Deplanque D, Staels B, et al. PPARs: a potential target for a disease-modifying strategy in stroke. CURR DRUG TARGETS. 2013;14(7):752-67.

5. Pereira MP, Hurtado O, Cardenas A, Bosca L, Castillo J, Davalos A, et al. Rosiglitazone and 15-deoxyDelta12,14-prostaglandin J2 cause potentneuroprotection after experimental stroke through noncompletely overlappingmechanisms. 2006. p. 218-29.

6. Lin T, Cheung W, Wu J, Chen J, Lin H, Chen J, et al. 15d-prostaglandin J2 protects brain from ischemia-reperfusion injury. Arterioscler Thromb Vasc Biol. 2006;26(3):481-7.

7. Ji S, Kronenberg G, Balkaya M, Farber K, Gertz K, Kettenmann H, et al. Acute neuroprotection by pioglitazone after mild brain ischemia without effect onlong-term outcome. EXP NEUROL. 2009;216(2):321-8. 'doi:'10.1016/j.expneurol.2008.12.007.

8. Kaundal RK, Sharma SS. GW1929: a nonthiazolidinedione PPARgamma agonist, ameliorates neurological damagein global cerebral ischemic-reperfusion injury through reduction in inflammation and DNA fragmentation. BEHAV BRAIN RES. 2011;216(2):606 - 12. 'doi:'10.1016/j.bbr.2010.09.001.

9. Culman J, Nguyen-Ngoc M, Glatz T, Gohlke P, Herdegen T, Zhao Y. Treatment of rats with pioglitazone in the reperfusion phase of focal cerebralischemia: a preclinical stroke trial. EXP NEUROL. 
2012;238(2):243 - 53. 'doi:'10.1016/j.expneurol.2012.09.003.

10. Zhao Y, Song J, Ma X, Zhang B, Li D, Pang H. Rosiglitazone ameliorates diffuse axonal injury by reducing loss of tau andup-regulating caveolin-1 expression. NEURAL REGEN RES. 2016;11(6):944 50. 'doi:'10.4103/1673-5374.184493.

11. Zhao Y, Wei X, Song J, Zhang M, Huang T, Qin J. Peroxisome Proliferator-Activated Receptor gamma Agonist Rosiglitazone ProtectsBlood-Brain Barrier Integrity Following Diffuse Axonal Injury by Decreasing theLevels of Inflammatory Mediators Through a Caveolin-1-Dependent Pathway. INFLAMMATION. 2019;42(3):841 - 56. 'doi:'10.1007/s10753-018-0940-2.

12. Huang Q, Zhong W, Hu Z, Tang X. A review of the role of cav-1 in neuropathology and neural recovery afterischemic stroke. J NEUROINFLAMM. 2018;15(1):348. 'doi:'10.1186/s12974-018-1387-y.

13. Choi K, Kim H, Park M, Kim J, Kim J, Cho K, et al. Regulation of Caveolin-1 Expression Determines Early Brain Edema AfterExperimental Focal Cerebral Ischemia. STROKE. 2016;47(5):1336-43. 'doi:'10.1161/STROKEAHA.116.013205.

14. Longa EZ, Weinstein PR, Carlson S, Cummins R. Reversible middle cerebral artery occlusion without craniectomy in rats. STROKE. 1989;20(1):84-91.

15. Li W, Chen Z, Chin I, Chen Z, Dai H. The Role of VE-cadherin in Blood-brain Barrier Integrity Under Central NervousSystem Pathological Conditions. CURR NEUROPHARMACOL. 2018;16(9):1375-84. 'doi:'10.2174/1570159X16666180222164809.

16. Neuhaus W, Piontek A, Protze J, Eichner M, Mahringer A, Subileau E, et al. Reversible opening of the blood-brain barrier by claudin-5-binding variants ofClostridium perfringens enterotoxin's claudinbinding domain. BIOMATERIALS. 2018;161:129 - 43. 'doi:'10.1016/j.biomaterials.2018.01.028.

17. Tang X, Zhong W, Tu Q, Ding B. NADPH oxidase mediates the expression of MMP-9 in cerebral tissue afterischemia-reperfusion damage. NEUROL RES. 2014;36(2):118 - 25. 'doi:'10.1179/1743132813Y.0000000266.

18. Anderson RG. The caveolae membrane system. ANNU REV BIOCHEM. 1998;67:199-225.

19. Liu P, Rudick M, Anderson RGW. Multiple functions of caveolin-1. J Biol Chem. 2002;277(44):412958.

20. Nguyen KCT, Cho KA. Versatile Functions of Caveolin-1 in Aging-related Diseases. Chonnam medical journal. 2017;53(1):28-36. 'doi:'10.4068/cmj.2017.53.1.28.

21. Ikezu T, Ueda H, Trapp BD, Nishiyama K, Sha JF, Volonte D, et al. Affinity-purification and characterization of caveolins from the brain:differential expression of caveolin- $1,-2$, and -3 in brain endothelial andastroglial cell types. BRAIN RES. 1998;804(2):177-92.

22. Virgintino D, Robertson D, Errede M, Benagiano V, Tauer U, Roncali L, et al. Expression of caveolin-1 in human brain microvessels. NEUROSCIENCE. 2002;115(1):145-52.

23. Stary CM, Tsutsumi YM, Patel PM, Head BP, Patel HH, Roth DM. Caveolins: targeting pro-survival signaling in the heart and brain. FRONT PHYSIOL. 2012;3:393. 'doi:'10.3389/fphys.2012.00393. 
24. Liu J, Wang LN. Peroxisome proliferator-activated receptor gamma agonists for preventing recurrent stroke and other vascular events in people with stroke or transient ischaemic attack. Cochrane Database Syst Rev. 2019;10:D10693. 'doi:'10.1002/14651858.CD010693.pub5.

25. Li Y, Zhu Z, Lu B, Huang T, Zhang Y, Zhou N, et al. Rosiglitazone ameliorates tissue plasminogen activator-induced brain hemorrhageafter stroke. CNS NEUROSCI THER. 2019;25(12):1343-52. 'doi:'10.1111/cns.13260.

26. Villapol S. Roles of Peroxisome Proliferator-Activated Receptor Gamma on Brain and Peripherallnflammation. CELL MOL NEUROBIOL. 2018;38(1):121 - 32. 'doi:'10.1007/s10571-0170554-5.

27. Shao Z, Liu Z. Neuroinflammation and neuronal autophagic death were suppressed via Rosiglitazone treatment: New evidence on neuroprotection in a rat model of global cerebral ischemia. J NEUROL SCI. 2015;349(1-2):65-71. 'doi:'10.1016/j.jns.2014.12.027.

28. Llaverias G, Vazquez-Carrera M, Sanchez RM, Noe V, Ciudad CJ, Laguna JC, et al. Rosiglitazone upregulates caveolin-1 expression in THP-1 cells through aPPAR-dependent mechanism. J LIPID RES. 2004;45(11):2015-24.

29. Tencer L, Burgermeister E, Ebert MP, Liscovitch M. Rosiglitazone induces caveolin-1 by PPARgammadependent and PPRE-independentmechanisms: the role of EGF receptor signaling and its effect on cancer cell drugresistance. ANTICANCER RES. 2008;28(2A):895-906.

30. Gu Y, Zheng G, Xu M, Li Y, Chen X, Zhu W, et al. Caveolin-1 regulates nitric oxide-mediated matrix metalloproteinases activity andblood-brain barrier permeability in focal cerebral ischemia and reperfusioninjury. J NEUROCHEM. 2012;120(1):147 - 56. 'doi:'10.1111/j.1471-4159.2011.07542.x.

31. Shen J, Ma S, Chan P, Lee W, Fung PC, Cheung RT, et al. Nitric oxide down-regulates caveolin-1 expression in rat brains during focal cerebral ischemia and reperfusion injury. J NEUROCHEM. 2006;96(4):1078-89. 'doi:'10.1111/j.1471-4159.2005.03589.x.

32. Bucci M, Gratton JP, Rudic RD, Acevedo L, Roviezzo F, Cirino G, et al. In vivo delivery of the caveolin-1 scaffolding domain inhibits nitric oxide synthesis and reduces inflammation. NAT MED. 2000;6(12):1362-7. 'doi:'10.1038/82176.

33. Sato Y, Sagami I, Shimizu T. Identification of caveolin-1-interacting sites in neuronal nitric-oxide synthase. Molecular mechanism for inhibition of NO formation. J BIOL CHEM. 2004;279(10):882736. 'doi:'10.1074/jbc.M310327200.

34. Zhang S, Liu Y, Zhao Z, Xue Y. Effects of green tea polyphenols on caveolin-1 of microvessel fragments in ratswith cerebral ischemia. NEUROL RES. 2010;32(9):963 - 70. 'doi:'10.1179/016164110X12700393823570.

35. Huang P, Zhou C, Qin-Hu, Liu Y, Hu B, Chang X, et al. Cerebralcare Granule(R) attenuates blood-brain barrier disruption after middlecerebral artery occlusion in rats. EXP NEUROL. 2012;237(2):453 - 63. 'doi:'10.1016/j.expneurol.2012.07.017.

36. Yang B, Xu J, Chang L, Miao Z, Heang D, Pu Y, et al. Cystatin C improves blood-brain barrier integrity after ischemic brain injury in mice. J NEUROCHEM. 2019. 'doi:'10.1111/jnc.14894. 


\section{Tables}

Table 1. Neurological deficit score of each group $(\mathrm{n}=24, \bar{x} \pm \mathrm{S})$

\begin{tabular}{ll}
\hline Group & Grade \\
\hline Sham group & 0 \\
MCAO group & $2.75 \pm 0.43^{*}$ \\
RSG group & $1.29 \pm 0.54^{* \#}$ \\
RSG+Daidzein group & $2.38 \pm 0.48^{* \# \boldsymbol{\Delta}}$ \\
\hline
\end{tabular}

${ }^{*}$ Compared with the sham group, $\mathrm{P}<0.05 ;{ }^{*}$ Compared with the MCAO group, $\mathrm{P}<0.05 ;{ }^{\wedge}$ Compared with the RSG group, $\mathrm{P}<0.05$

\section{Figures}




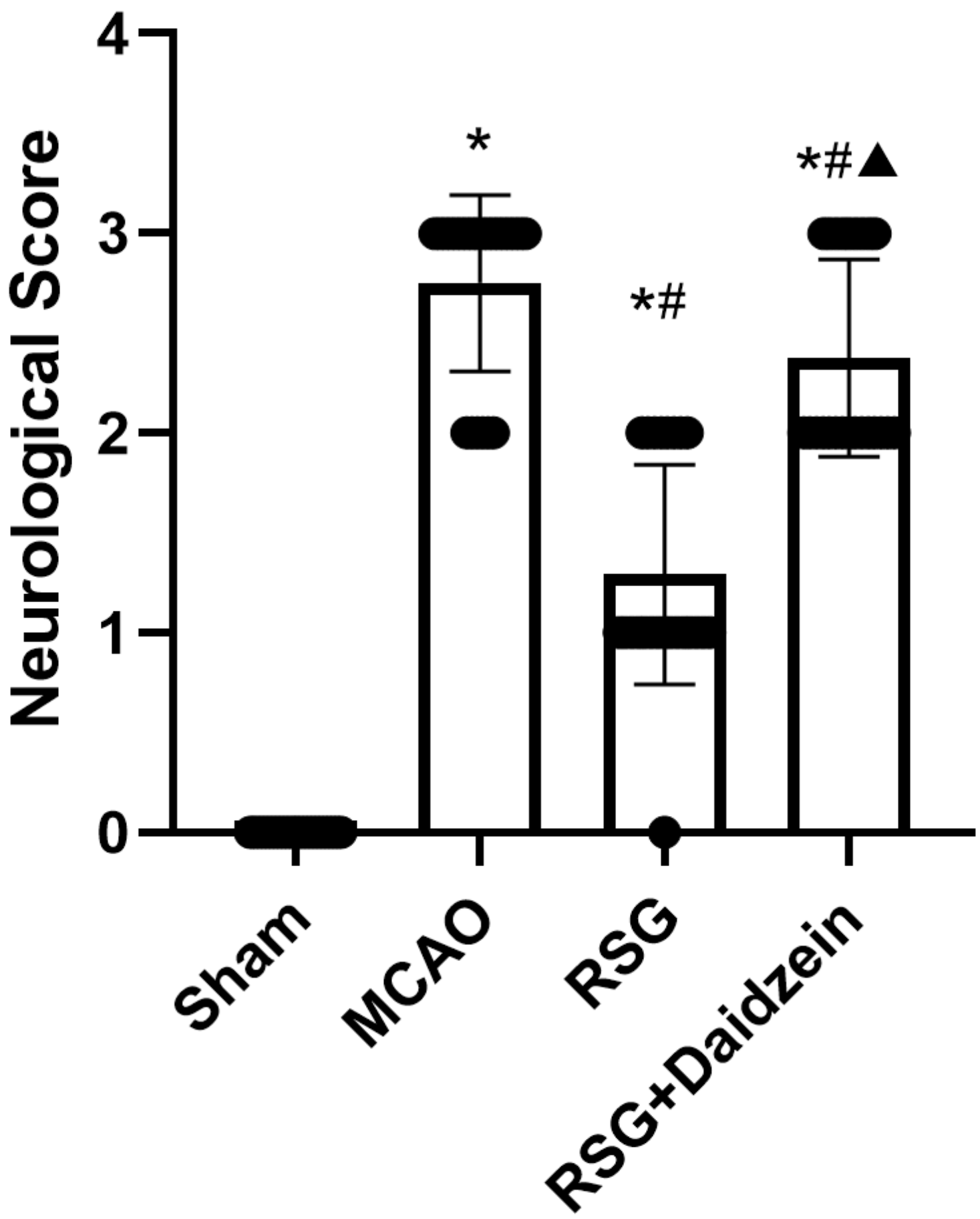

Figure 1

Neurological deficit score of each group. ( $\mathrm{n}=24 ;{ }^{*}$ Compared with the sham group, $\mathrm{P}<0.05$; \# Compared with the MCAO group, $\mathrm{P}<0.05 ; \boldsymbol{\Delta}$ Compared with the RSG group, $\mathrm{P}<0.05)$. Data are presented as the mean \pm SD. 
a

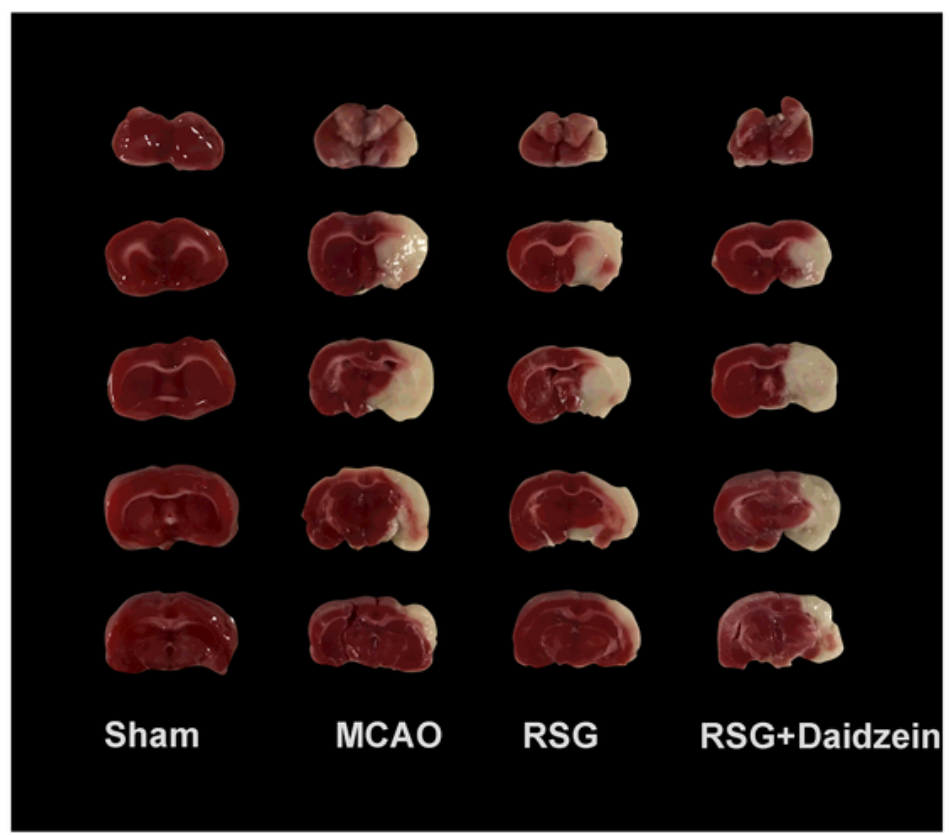

b

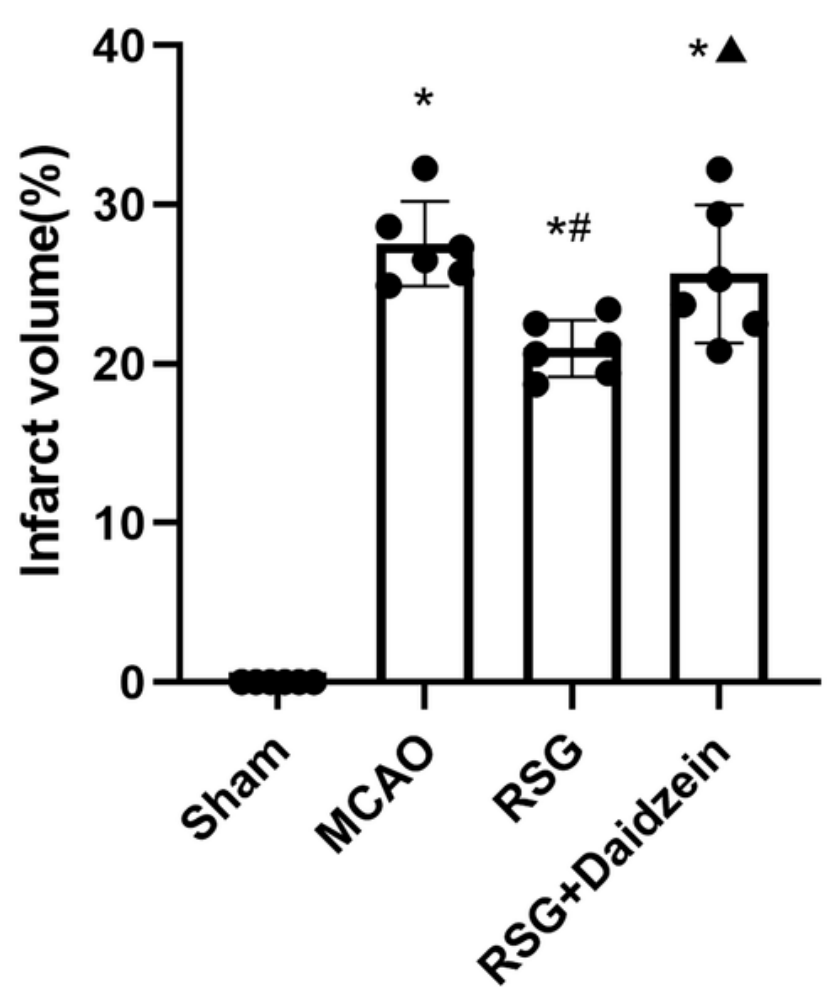

Figure 2

Effects of RSG and RSG + Daidzin (caveolin-1 antagonists) on the infarct volume of rats. a TTC staining. b Relative infarct volume. $(n=6 ; * P<0.05$, versus the sham group; $\# P<0.05$, versus the MCAO group; $\boldsymbol{\Delta} P$ $<0.05$, versus the RSG group;). Data are presented as the mean \pm SD. 
a

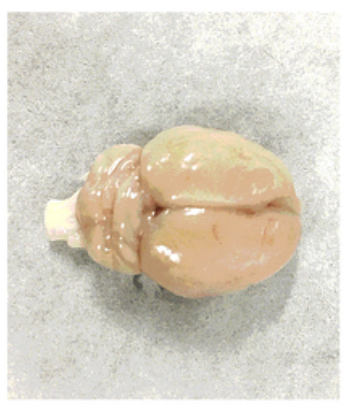

Sham

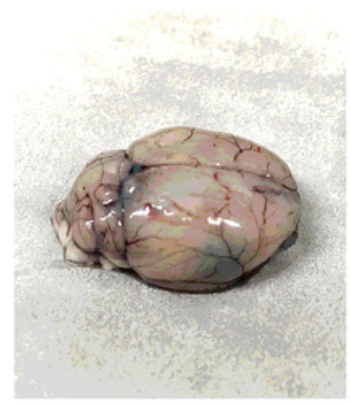

MCAO

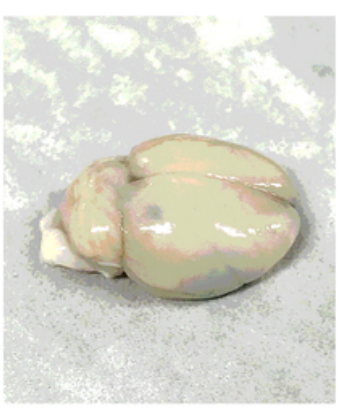

RSG

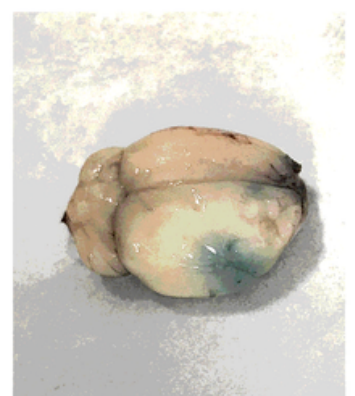

RSG+Daidzein

b

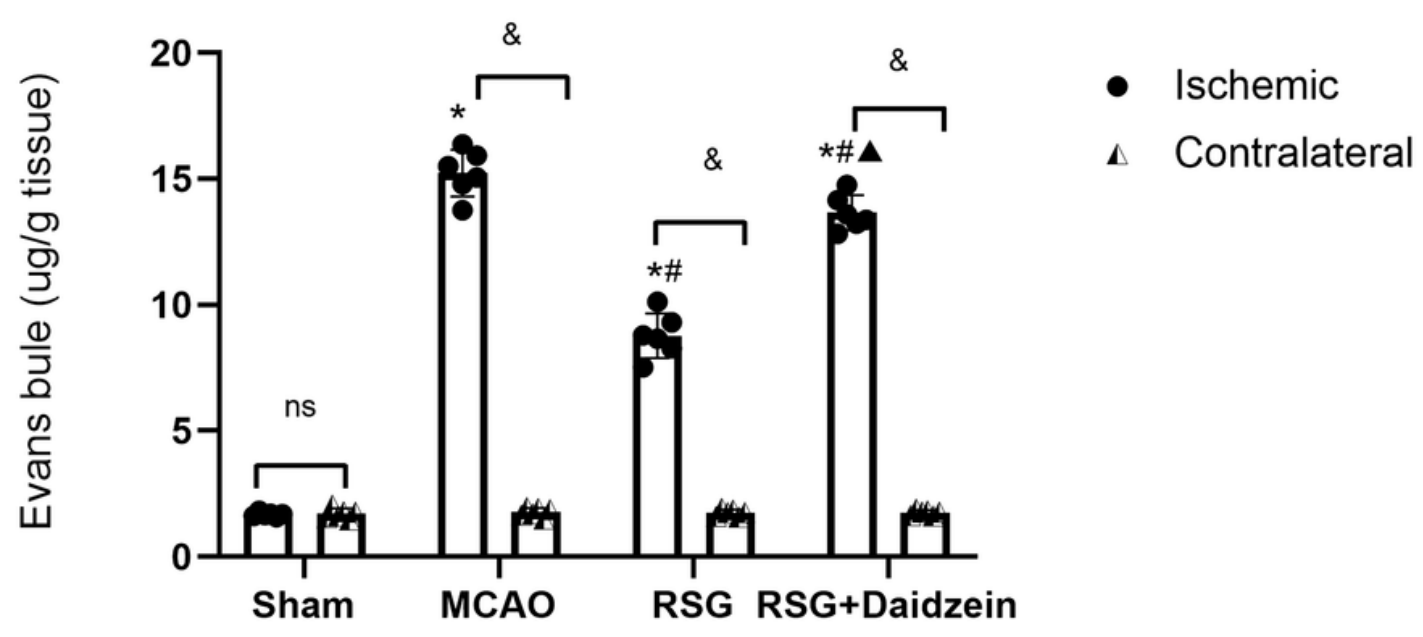

Figure 3

Effects of RSG and RSG + Daidzin (caveolin-1 antagonists) on the permeability of the BBB following cerebral ischemia. a Photos representing Evans blue leakage in different groups. b The statistical analysis of the Evans blue diffusion $(n=6 ; * P<0.05$, versus the sham group; $\# P<0.05$, versus the MCAO group; $\Delta P<0.05$, versus the RSG group; \&P $<0.05$, versus the contralateral brain). Data are presented as the mean $\pm S D$. 
(a)

\section{Caveolin1}

MMP-9

Occludin

GAPDH
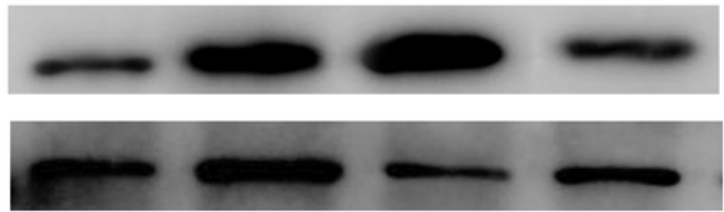

(c)

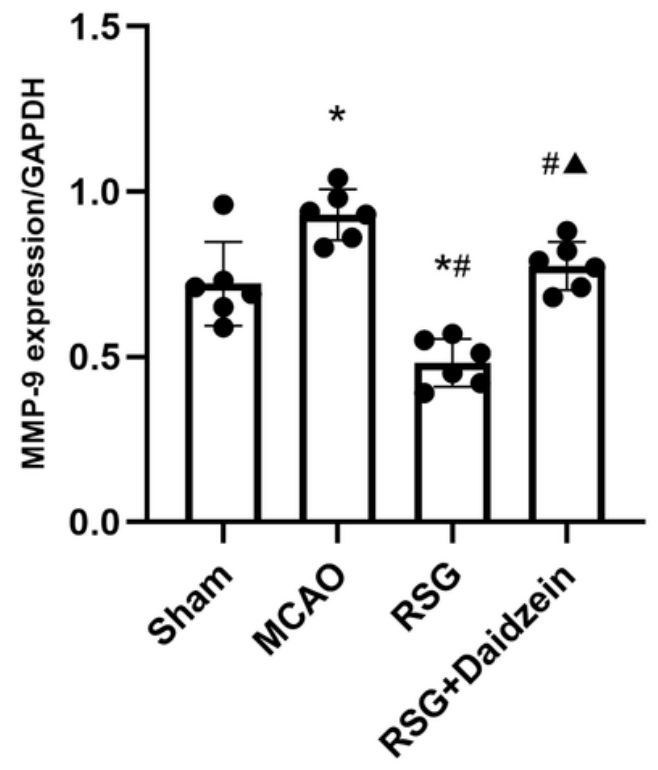

(b)

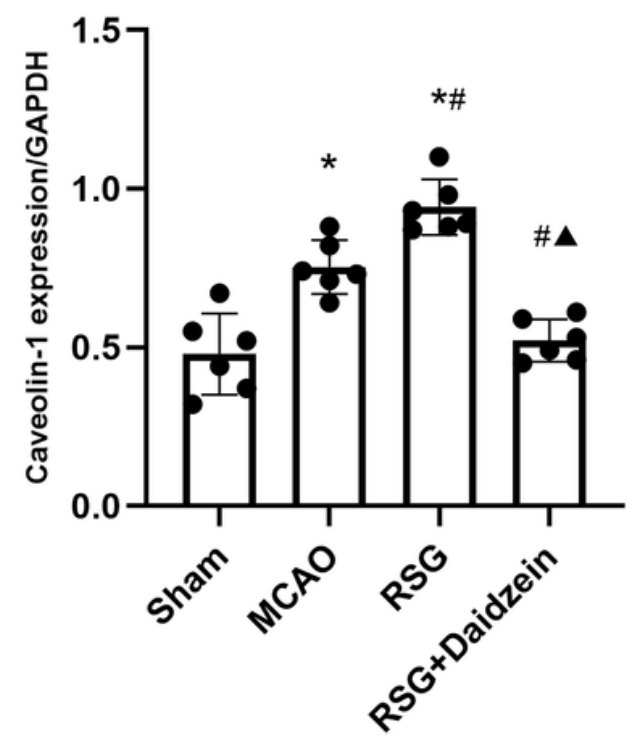

(d)

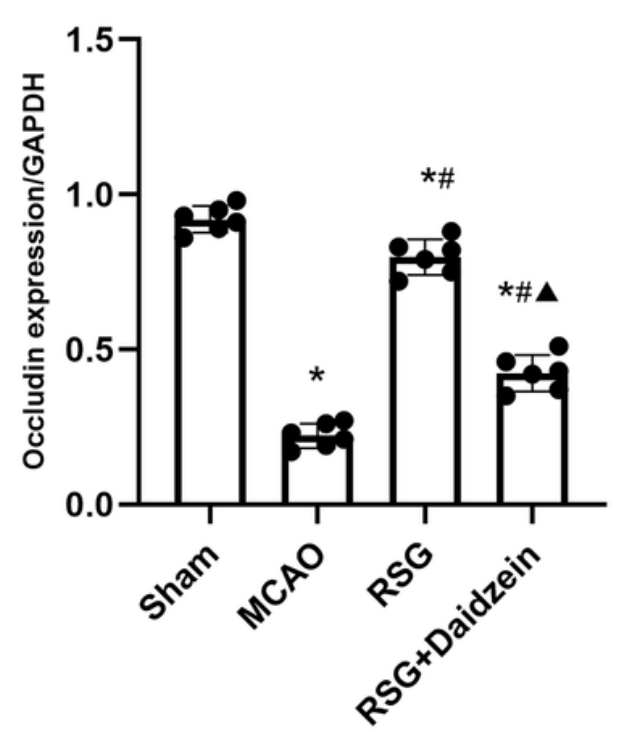

Figure 4

The levels of expression of MMP-9, caveolin-1, and occludin determined by Western blot. GAPDH was used as an internal control. The bar graphs show the statistical analysis of the expression levels of of MMP-9, caveolin-1, and occludin in the different experimental groups. All data are presented as the mean $\pm S D\left(n=6 ;{ }^{*}<0.05\right.$, versus the sham group; $\# P<0.05$, versus the MCAO group; $\Delta P<0.05$, versus the RSG group). 

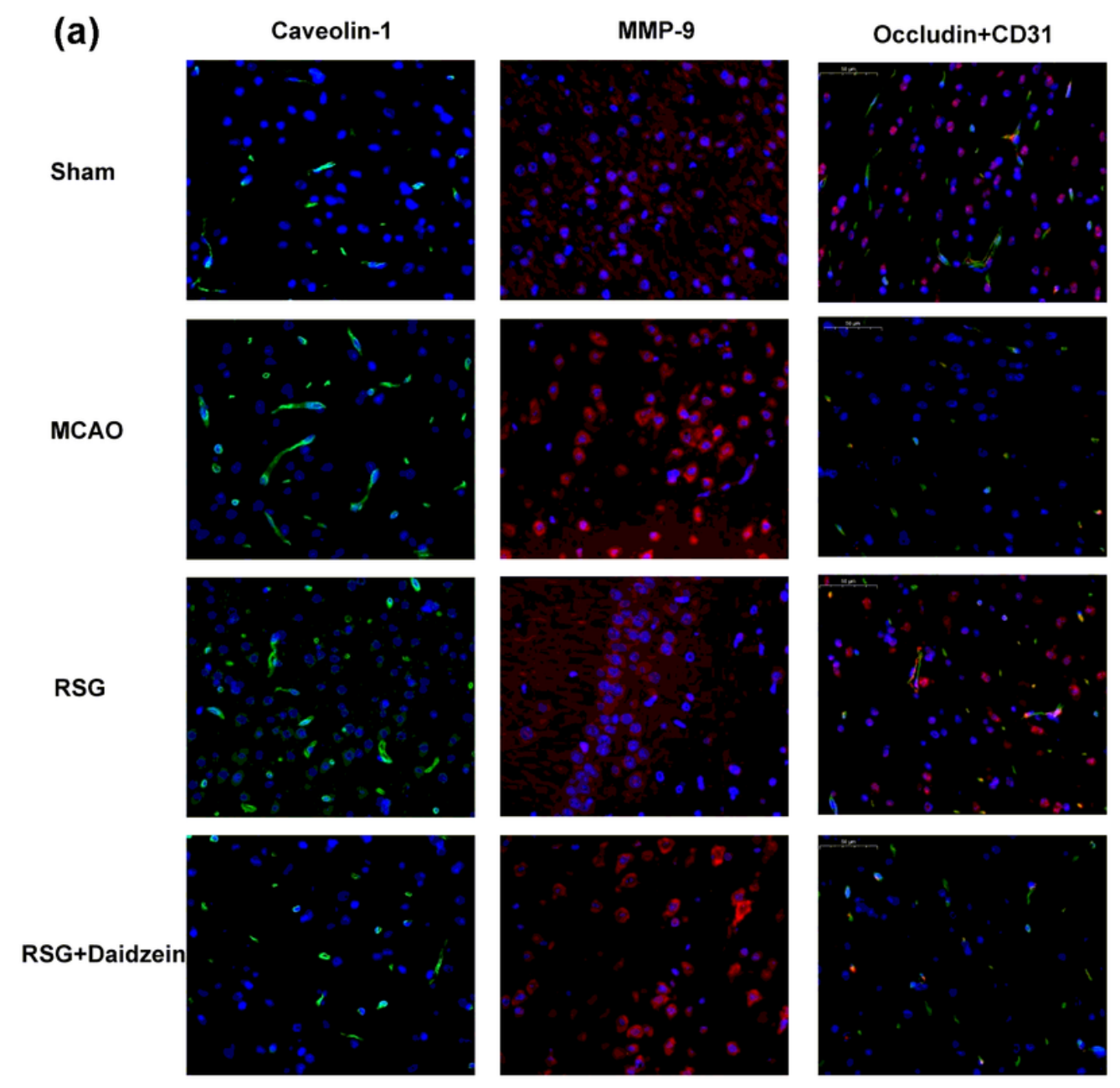

(b)

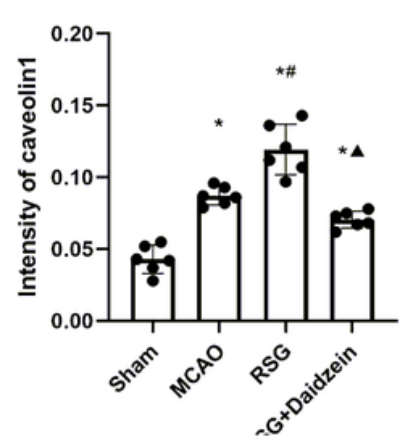

(c)

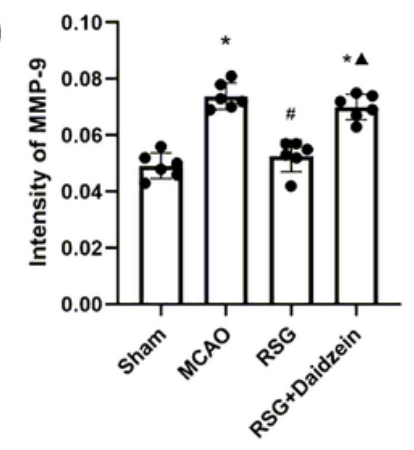

(d)

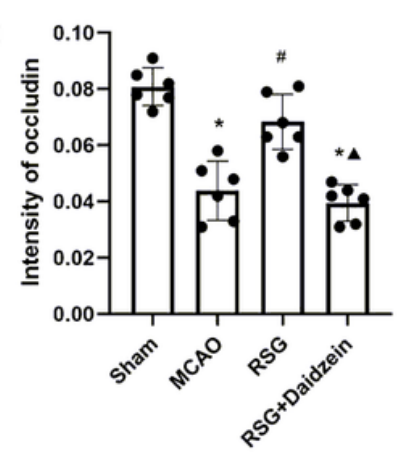

\section{Figure 5}

The expression levels of MMP-9, caveolin-1, and occludin assessed by immunofluorescence $(\times 400$ magnification, $n=6$ ). a Representative immunofluorescence images of caveolin-1, MMP-9, and CD31(red) and occludin(green) double-immunostaining in the rats.b Statistical analysis of caveolin-1. c Statistical analysis of MMP-9. d Statistical analysis of occludin. ${ }^{*} \mathrm{P}<0.05$, versus the sham group; \#P $<0.05$, versus the MCAO group; $\triangle P<0.05$, versus the RSG group

\section{Supplementary Files}

This is a list of supplementary files associated with this preprint. Click to download.

- Onlinefigures1.png

- Onlinefigures2.png

- OnlinefigureS3.png 